\title{
A New Analytical Approach for the Velocity Field in Rolling Processes and Its Application in Through-Thickness Texture Prediction
}

\author{
KOEN DECROOS, JURIJ SIDOR, and MARC SEEFELDT
}

\begin{abstract}
A new analytical model is presented that expresses kinematically admissible velocity fields in rolling processes. Opposed to conventional streamline approaches, the current model does not force the material to flow along the prescribed lines, but introduces a new coordinate that is constant over these lines, to prescribe a fixed component of the velocity in the rolling direction as a function of that coordinate and the coordinate along the rolling direction. The interaction between the rolls and the surface is incorporated in the model via two scalar parameters which depend on the friction conditions between the roll and the sheet, and the properties of rolled material. The scalar parameters can be tuned with experimental observations of deformation flow across the thickness. The modelled material flow does not reveal significant deviation from the one calculated by streamlines. The obtained analytical expressions for the velocity gradient tensor components combined with polycrystal plasticity models enables the prediction of the through-thickness texture evolution for various friction conditions.
\end{abstract}

DOI: $10.1007 / \mathrm{s} 11661-013-2021-3$

(C) The Minerals, Metals \& Materials Society and ASM International 2013

\section{INTRODUCTION}

ON single crystal level, plastic deformation occurs on slip systems, which consist of a slip plane and a slip direction. ${ }^{[1]}$ When a large plastic deformation occurs in a polycrystalline aggregate, each particular crystal rotates in order to maintain compatibility with its surrounding and as a result a specific crystallographic orientation distribution arises called texture ${ }^{[1]}$ As the single crystal's plastic behavior is highly anisotropic, a textured material has in general an anisotropic plastic behavior. Knowledge of the texture and anisotropy is important since it influences the macroscopic deformation behavior such as formability. A more sophisticated material law could be used as an input in various numerical and analytical approaches such as the finite element (FE) based simulations, ${ }^{[2]}$ the slab method, ${ }^{[3]}$ the slip-line method ${ }^{[4]}$ and the upper bound method ${ }^{[5-7]}$ to calculate various technological outputs of the rolling process.

In order to predict the plastic behavior of a polycrystalline material, several models have been developed. These models could be subdivided into two types, namely the computationally costly crystal plasticity finite element models (CPFEM) ${ }^{[8,9]}$ and statistical mes-

KOEN DECROOS, Post-doctoral Researcher, and MARC SEEFELDT, Professor, are with the Department of Metallurgy and Materials Engineering, Faculty of Engineering, Catholic University of Leuven, Heverlee, 3001 Leuven, Belgium. Contact e-mail: koen.Decroos@ gmail.com, koenraad.decroos@mtm.kuleuven.be JURIJ SIDOR, Research Assistant, is with the Department of Materials Science and Engineering, Faculty of Engineering and Architecture, Ghent University, Zwijnaarde, 9052 Gent, Belgium.

Manuscript submitted January 10, 2013.

Article published online November 7, 2013 oscopic polycrystal plasticity models. In polycrystal plasticity models, the macroscopic velocity gradient tensor is imposed whereas the stresses and strains in the grains, as well as the macroscopic stress, are obtained based on specific assumptions. Particular examples are the Taylor ${ }^{10]}$ model, the visco-plastic self consistent model, ${ }^{[11]}$ the Alamel model,$^{[12]}$ and the GIA model ${ }^{[13]}$ An overview of these models is given by Qie et al. ${ }^{[14]}$

Numerous literature sources report on the calculation of the velocity gradient tensor coupled with polycrystal plasticity models. ${ }^{[15-18]}$ Choi et al. ${ }^{[15]}$ used the FE method to calculate the local strain evolution. Van Houtte et al. used an incremental texture updating procedure in a FE model of a cup drawing process. ${ }^{[1]}$ Segurado et al. ${ }^{[17]}$ reported on a multiscale model to embed the VPSC model in an implicit FE model of sheet rolling. Engler et al. ${ }^{[18]}$ imposed a sine-shaped 31 and 13 shear (where 1 represents the rolling direction, and 3 the sheet's normal direction) in the top layers to implement the shear between the rolls and the plate's surface. Alternatively, the deformation flow across the thickness in rolled materials is described by analytical flow functions. Both two and three dimensional functions were proposed to construct the kinematically admissible strain velocity fields. ${ }^{[19-26]}$ The single and dual-stream functions are constructed in a way that the velocity vector at a point in the deformation zone is the tangential vector to the flowline passing through this point. It is assumed that the material flows along prescribed trajectories called flowlines or streamlines. In the streamline approaches, the components of velocity gradient tensor are expressed as partial derivates of the velocity field along a particular direction. A kinematically admissible velocity ensures the flow of a material along the flowlines and also fulfills the material's 
incompressibility condition. The flowlines are represented by a parametric family of curves, and could be expressed as parabolic, ${ }^{[25]}$ circular, elliptical, ${ }^{[19]}$ or a complex shape function. ${ }^{[23,26]}$ Since the described methods deal with analytical functions, they are able to find analytical expressions for the components of the velocity gradient tensor. The non uniform velocity gradient field across the thickness of a rolled sheet explains the through-thickness texture gradient, as reported in numerous literature sources. ${ }^{[15,18,27]}$ The flow line models are capable of reproducing the shear component in the rolling plane by assigning heterogeneous velocity distribution at the end of the flowline. However, current state of the art does not contain a streamline formulation with a continuous velocity field homogeneous outside of the deformation zone, and which can alter when different frictional conditions applied between the rolls and the plate's surface. Different frictional conditions lead to various deformation flow across the thickness, which can be visualized by considering the deformation after rolling of an initially rectangular grid put on the plate, the so called grid pattern technique. ${ }^{[28,29]}$

The goal of the present work is to predict the through-thickness deformation in rolled materials and to calculate the corresponding texture development. The strain fields at different through thickness planes are calculated by the presented analytical approach, and are used as an input for a crystal plasticity model that calculates textures. The analytical approach for the velocity field, although not fulfilling stress equilibrium, offers the advantage over the FE method that the calculation procedure is drastically faster.

\section{SPECIMEN AND PROCESS}

In this work, the cold rolling process of a plate made of DC04 single phase ferritic steel is studied. The process and rolling parameters are schematically shown in Figure 1. The parameters are the initial sheet thickness $2 e(\mathrm{~mm})$, the final sheet thickness $2 s(\mathrm{~mm})$, the roll's radius $R(\mathrm{~mm})$, and the roll's angular velocity $\omega(\mathrm{rad} / \mathrm{s})$.

In this paper, a typical cold rolling process with a significant thickness reduction has been studied. A sheet's thickness reduction from $2 e=1$ to $2 s=0.6 \mathrm{~mm}$ is considered. The rolling mills with a radius of $12 \mathrm{~cm}$ rotate with an angular velocity of 10 rotations per minute. Different frictional conditions between the rolling mills and the plate have been considered. The rolling process is assumed to be in the steady state condition. Due to the symmetry of the problem in the horizontal plane $z=0$, it is sufficient to consider only one half of the sheet.

The texture evolution across the thickness of rolled DC04 steel was predicted with the viscoplastic selfconsistent (VPSC) model $^{[11]}$ with an affine linearization scheme. Since the investigated material has a body centered cubic crystal structure, 48 possible slip systems of three families were taken into account: $\{112\}\langle 111\rangle$, $\{110\}\langle 111\rangle$, and $\{123\}\langle 111\rangle$.

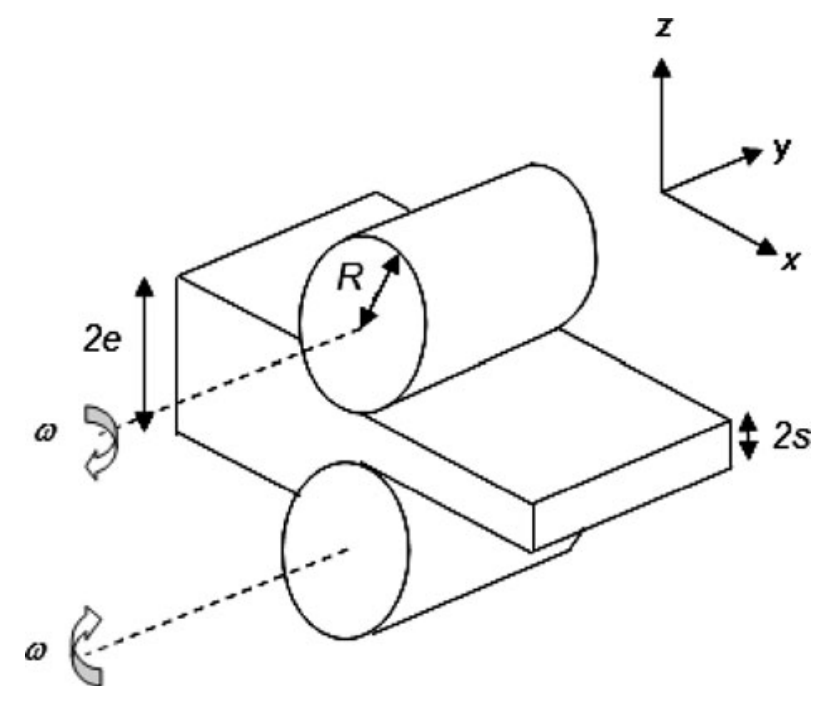

Fig. 1-Schematic representation of the rolling process and the definition of the rolling parameters.

\section{MATHEMATICAL PROCEDURE}

\section{A. Streamline Functions}

In two-dimensional flowline or streamline modeling of rolling processes, the material flow in the steady state condition is assumed to follow prescribed lines. Hereby the streamline is defined as a one parameter family of curves, where designating a specific value to the parameter variable corresponds to defining one streamline, determined by a relationship between $x$ and $z$. In general, a plane strain streamline is described by Eq. [1], where $z_{\mathrm{s}}$ is the streamline parameter varying from 0 to 1 , and $\phi$ represents the relationship between $x$ and $z$ for a given value of $z_{\mathrm{s}}$

$$
z_{\mathrm{s}}=\phi(x, z)
$$

The function $\phi(x, z)$ determines the nature of the streamline. In this paper, the function $\phi(x, z)$ is defined by Eq. [2] based on a recently reported stream function $^{[26]}$

$$
\begin{aligned}
& \phi(x, z)=z \varsigma(x) \\
& = \begin{cases}z \frac{1}{e}\left[1+\left(\frac{s}{e}+\left(1-\frac{s}{e}\right)\left(\frac{d-x}{d}\right)^{2.1}\right)^{-m}\right]^{\frac{1}{m}}, & x \leq d \\
z \frac{1}{e}\left[1+\left(\frac{s}{e}\right)^{-m}\right]^{\frac{1}{m}} \approx z \frac{1}{s}, & x>d\end{cases}
\end{aligned}
$$

where $d=R \sin (\theta)$ and $\theta=\cos ^{-1}(R+s-e) / R$.

The principle of streamlines and the meaning of the geometric parameters of Eq. [2] are shown in Figure 2.

Equation [2] defines the function $\phi(x, z)$ prior, during, and after the deformation zone. The introduction of the parameter $m$, which is assumed to be 50 in this paper, guarantees a continuous first and second derivative of the function $\varsigma(x)$ of Eq. [2] in $x=0 .{ }^{[26]}$ The exponent of 2.1 in Eq. [2] has been chosen such that both the function in the deformation zone is quasi-parabolic, and 


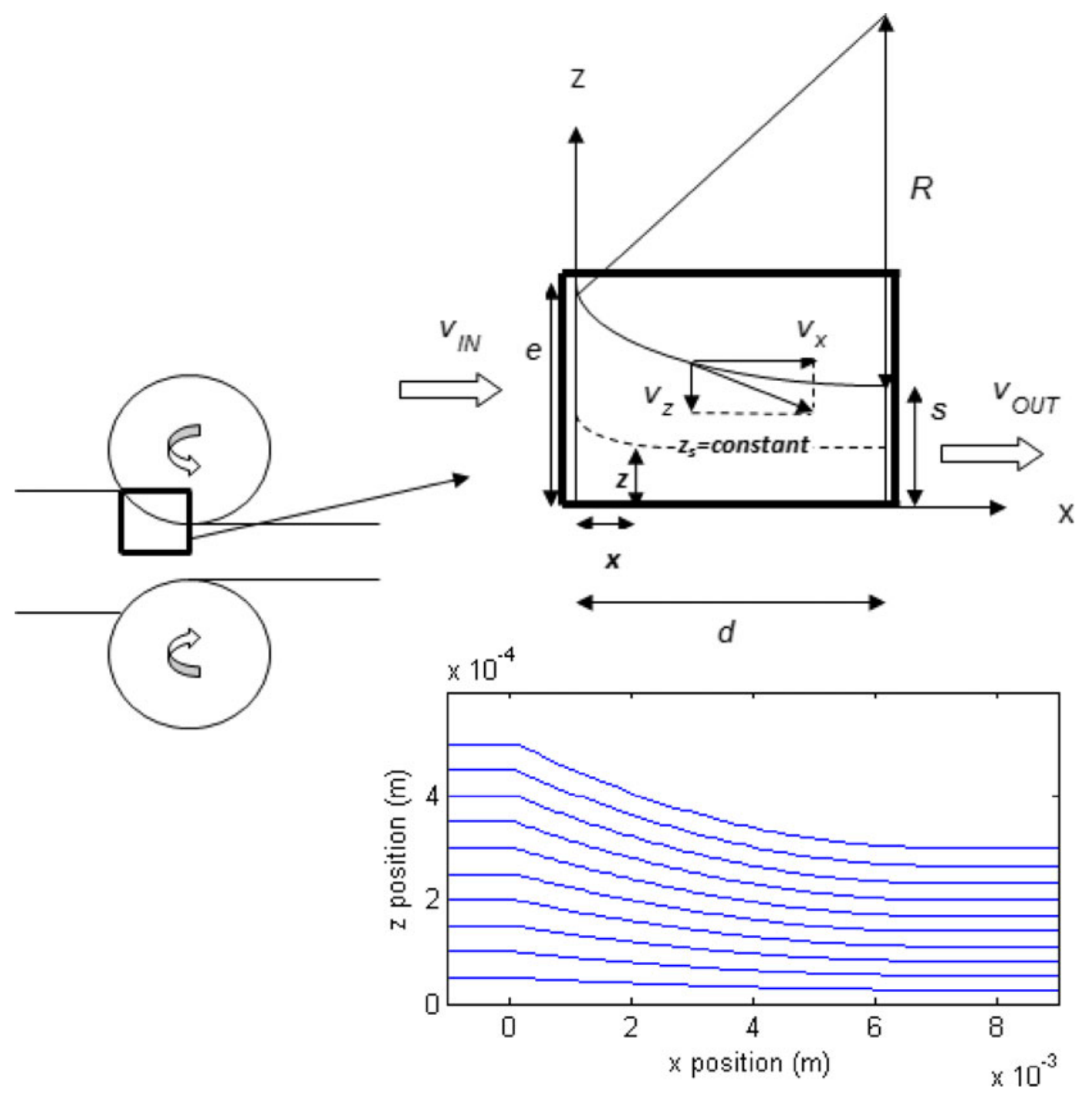

Fig. 2-The principle of streamlines according to Eqs. [1] and [2]. Lines for values of $z_{\mathrm{s}}$ going from 0 to 1 are plotted.

at $x=d$ the first and second derivatives of $\varsigma(x)$ are continuous functions.

Equation [3] ensures that material points follow the streamlines determined by Eq. [1]:

$$
\begin{aligned}
& v_{x}=\lambda(x, z) \frac{\partial \phi(x, z)}{\partial z} \\
& v_{z}=-\lambda(x, z) \frac{\partial \phi(x, z)}{\partial x}
\end{aligned}
$$

Hereby $\lambda(x, z)$ is an arbitrary function of $x$ and $z$.

In order to have a kinematically admissible velocity field, at any time the incompressibility condition of Eq. [4] must be fulfilled.

$$
\dot{\varepsilon}_{x x}+\dot{\varepsilon}_{z z}=\frac{\partial}{\partial x} v_{x}(x, z)+\frac{\partial}{\partial z} v_{z}(x, z)=0
$$

Alternatively, it is also possible to invert relationship 1 and express $z$ as a function of $x$, and $z_{\mathrm{s}}$, as in Eq. [5].

$$
z=\varphi\left(x, z_{\mathrm{s}}\right)
$$

Consequently, every function of $x$ and $z$ can be expressed as a function of $x$ and $z_{\mathrm{s}}$. As a consequence functions of $x$ and $z_{\mathrm{s}}$ will be denoted by means of an asterisk. For example, combining Eqs. [5] and [3] results in Eq. [6]:

$$
\begin{aligned}
& v_{x}(x, z)=v_{x}\left(x, \varphi\left(x, z_{\mathrm{s}}\right)\right)=v_{x}^{*}\left(x, z_{\mathrm{s}}\right)=\lambda^{*}\left(x, z_{\mathrm{s}}\right) \frac{\partial \phi(x, z)}{\partial z} \\
& v_{z}(x, z)=v_{z}\left(x, \varphi\left(x, z_{\mathrm{s}}\right)\right)=v_{z}^{*}\left(x, z_{\mathrm{s}}\right)=-\lambda^{*}\left(x, z_{\mathrm{s}}\right) \frac{\partial \phi(x, z)}{\partial x}
\end{aligned}
$$

Substitution of Eq. [6] in Eq. [4] results in Eq. [7] 


$$
\begin{aligned}
& \frac{\partial}{\partial z} \phi(x, z) \frac{\partial}{\partial x} \lambda^{*}\left(x, z_{\mathrm{s}}\right)+\lambda^{*}\left(x, z_{\mathrm{s}}\right) \frac{\partial^{2} \phi(x, z)}{\partial x \partial z} \\
& \quad+\left[\frac{\partial}{\partial z_{\mathrm{s}}} \lambda^{*}\left(x, z_{\mathrm{s}}\right)\right] \frac{\partial z_{\mathrm{s}}}{\partial x} \frac{\partial}{\partial z} \phi(x, z)-\lambda^{*}\left(x, z_{\mathrm{s}}\right) \frac{\partial^{2} \phi(x, z)}{\partial z \partial x} \\
& \quad-\left[\frac{\partial}{\partial z_{\mathrm{s}}} \lambda^{*}\left(x, z_{\mathrm{s}}\right)\right] \frac{\partial z_{\mathrm{s}}}{\partial z} \frac{\partial}{\partial x} \phi(x, z)=0
\end{aligned}
$$

Elaboration of Eq. [7] results in Eq. [8]

$$
\frac{\partial}{\partial x} \lambda^{*}\left(x, z_{\mathrm{s}}\right)=0 .
$$

Equation [8] implies that in order to fulfill both the incompressibility and the condition of the material flow along the predefined streamlines, the function $\lambda^{*}\left(x, z_{\mathrm{s}}\right)$ should be dependent only on $z_{\mathrm{s}}$. Assuming streamlines according to Eq. [2], that condition yields in the expression of the velocity field in Eq. [9]

$$
\begin{aligned}
& v_{x}^{*}\left(x, z_{\mathrm{s}}\right)=\lambda^{*}\left(z_{\mathrm{s}}\right) \zeta(x) \\
& v_{z}^{*}\left(x, z_{\mathrm{s}}\right)=-\lambda^{*}\left(z_{\mathrm{s}}\right) \frac{z_{\mathrm{s}}}{\zeta(x)} \frac{d}{\mathrm{~d} x} \zeta(x) .
\end{aligned}
$$

The $x$-component of the velocity is expressed as a product of a factor depending only on the variable $z_{\mathrm{s}}$, and a factor depending only on the variable $x$. A major disadvantage of the streamline approach is that in order to have a constant $x$-component of the velocity field over the thickness (for different values of $z_{\mathrm{s}}$ ) for certain values for $x$, as would be necessary if the deformation zone were approximated by the box in Figure 2, and one would want a uniform entrance and exit velocity over the sheet's thickness outside the deformation zone, the function $\lambda^{*}$ should be independent of $z_{\mathrm{s}}$. That is due to fact that the contributions of $x$ and $z_{\mathrm{s}}$ are separated as a product of factors, which consequently would impose the function $\lambda^{*}$ to be a constant. In that case, there would be a conflict with the demand to have various rates of deformation flow across the thickness along the $x$-direction, which would be necessary in order to capture the effect of the higher velocity near the contact surface compared to the middle of the plate. That would explicitly be necessary if one would like to incorporate the effect of different frictional conditions between the rolls and the plate. In that case there clearly should be a dependence of $z_{\mathrm{s}}$. It could be concluded that previously described method fails in capturing both a uniform velocity outside the deformation zone and a through thickness variation of the $x$-component of the velocity within the deformation zone.

\section{B. A New Model}

\section{Velocity field}

In the current approach the material is not longer forced to follow the streamlines determined by $z_{\mathrm{s}}=$ constant, whereas $z_{\mathrm{s}}$ is now considered as a new coordinate, defined by Eq. [1]. However, the $x$-component of the velocity is prescribed in the specimen, depending on the $x$ coordinate, and the $z_{\mathrm{s}}$ coordinate, and is a function of the friction conditions. In this approach, except for the mid-thickness part and the surface of the plate, the material streamlines are initially unknown, contrary to the streamline models. The deformation zone is considered to be delimited by the vertical lines at $x=0$ and $x=d$, cf. Figure 2. The current research aims to present a kinematically admissible velocity field, which fulfills following conditions:

(i) At the entrance and the exit of the rolling process, the velocity is uniform across the thickness.

(ii) The incompressibility condition is fulfilled at all points in the material.

(iii) In the middle $\left(z_{\mathrm{s}}=0\right)$ and the surface $\left(z_{\mathrm{s}}=1\right)$ of the plate, the material follows the streamlines $\left(z_{\mathrm{s}}=\right.$ constant $)$ as defined by Eqs. [1] and [2].

iv) At the surface, the velocity field is prescribed with a parameter that is directly linked to the friction conditions between the rolls and the plate.

The $x$-component of the velocity field is assumed to be a function of $x$ and $z_{\mathrm{s}}$ in the deformation zone:

$$
\begin{aligned}
v_{x}^{*}\left(x, z_{\mathrm{s}}\right) & =f_{1}(x)\left(1-z_{\mathrm{s}}^{n}\right)+f_{2}(x) z_{\mathrm{s}}^{n} \\
& =\left[f_{2}(x)-f_{1}(x)\right] z_{\mathrm{s}}^{n}+f_{1}(x) .
\end{aligned}
$$

In the middle of the plate, the coordinate $z_{\mathrm{s}}$ equals 0 , and the $x$-component of the velocity is given by the function $f_{1}(x)$. At the surface of the plate, $z_{\mathrm{s}}$ equals 1 , and the $x$-component of the velocity is given by the function $f_{2}(x)$. The dependency of the velocity of the through thickness coordinate $z_{\mathrm{s}}$ is an $n$th power law. The parameter $n$, which should be greater than 1 in order to impose that the variation of the velocity is the highest near the surface, controls the difference in velocity of deformation flow across the thickness due to friction. The parameter $n$ is material and temperature dependent.

The boundary condition (i) is translated mathematically into Eq. [11] as follows:

$$
\begin{aligned}
& f_{1}(x=0)=f_{2}(x=0)=v_{\mathrm{IN}} \\
& f_{1}(x=d)=f_{2}(x=d)=v_{\mathrm{OUT}}
\end{aligned}
$$

The condition (ii) is the incompressibility condition of Eq. [4]. The first term of Eq. [4] results in Eq. [12] if one substitutes the proposed expression of Eq. [10]:

$$
\begin{aligned}
\frac{\partial}{\partial x} v_{x}(x, z)= & \frac{\partial}{\partial x} v_{x}^{*}\left(x, z_{\mathrm{s}}\right)+\frac{\partial}{\partial z_{\mathrm{s}}} v_{x}^{*}\left(x, z_{\mathrm{s}}\right) \frac{\partial z_{\mathrm{s}}}{\partial x} \\
& =\left(\frac{d}{\mathrm{~d} x} f_{2}(x)-\frac{d}{\mathrm{~d} x} f_{1}(x)\right) z_{\mathrm{s}}^{n}+\frac{d}{\mathrm{~d} x} f_{1}(x) \\
& +\left[f_{2}(x)-f_{1}(x)\right] n z_{\mathrm{s}}^{n-1} \frac{\partial z_{\mathrm{s}}}{\partial x}
\end{aligned}
$$

The second term of Eq. [4] is given by Eq. [13]:

$$
\frac{\partial}{\partial z} v_{z}(x, z)=\frac{\partial}{\partial z_{\mathrm{s}}} v_{z}^{*}\left(x, z_{\mathrm{s}}\right) \frac{\partial z_{\mathrm{s}}}{\partial z} .
$$


Substituting the first and second terms of the incompressibility condition of Eq. [4] by formulas 12 and 13 and considering the expression for $z_{\mathrm{s}}$ (Eqs. [1] and [2]) results in the partial differential Eq. [14] in the $z$ component of the velocity expressed in terms of $x$ and $z_{\mathrm{s}}$ :

$$
\begin{aligned}
\frac{\partial}{\partial z} v_{z}(x, z)= & -\frac{1}{\varsigma(x)}\left[\left[\frac{d}{\mathrm{~d} x} f_{2}(x)-\frac{d}{\mathrm{~d} x} f_{1}(x)\right] z_{\mathrm{s}}^{n}\right. \\
& \left.+\frac{d}{\mathrm{~d} x} f_{1}(x)+\left[f_{2}(x)-f_{1}(x)\right] n \frac{z_{\mathrm{s}}^{n}}{\varsigma(x)} \frac{d}{\mathrm{~d} x} \varsigma(x)\right]
\end{aligned}
$$

Integration of Eq. [14] yields to the Eq. [15]:

$$
\begin{aligned}
& v_{z}^{*}\left(x, z_{\mathrm{s}}\right) \\
& \quad=-\frac{1}{\varsigma(x)}\left[\begin{array}{l}
\left(\frac{d}{\mathrm{~d} x} f_{2}(x)-\frac{d}{\mathrm{~d} x} f_{1}(x)\right) \frac{z_{\mathrm{s}}^{n+1}}{n+1}+\frac{d}{\mathrm{~d} x} f_{1}(x) z_{\mathrm{s}} \\
+n\left(f_{2}(x)-f_{1}(x)\right) \frac{z_{\mathrm{s}}^{n+1}}{n+1} \frac{1}{\varsigma(x)} \frac{d}{\mathrm{~d} x} \varsigma(x)
\end{array}\right] \\
& +g(x) .
\end{aligned}
$$

Thereby the function $g(x)$ is an arbitrary function of $x$, which is determined by means of boundary conditions. According to the boundary condition (iii), the $z$-component of the velocity is 0 if $z_{\mathrm{s}}$ equals to 0 , for all values of $x$, therefore, the function $g(x)$ for this condition is equal to 0 .

Condition (iii) at the outer flowline states that the material should follow the flowline for $z_{\mathrm{s}}=1$. Therefore, for all values of $x$, the condition of Eq. [3] results in Eq. [16]:

$$
\frac{v_{x}^{*}(x, 1)}{v_{z}^{*}(x, 1)}=-\frac{\frac{\partial z_{\mathrm{s}}}{\partial z}}{\frac{\partial z_{\mathrm{s}}}{\partial x}} .
$$

Considering Eqs. [1] and [2] for $z_{\mathrm{s}}$, and the expressions 10 and 15 for the velocities, Eq. [16] results in differential Eq. [17]. In order not to overload the notations, the arguments of the functions are no longer written from here, and a derivative of a function of one argument is noted with an accent (')

$$
\frac{\varsigma^{\prime}}{\varsigma^{2}}=-\frac{1}{\varsigma} \frac{\frac{1}{n+1}\left[\left(f_{2}^{\prime}-f_{1}^{\prime}\right)+n\left(f_{2}-f_{1}\right) \frac{\varsigma^{\prime}}{\varsigma}\right]+f_{1}^{\prime}}{f_{2}} .
$$

Further elaboration of Eq. [17] results in Eq. [18].

$$
\begin{aligned}
f_{2} \frac{\varsigma^{\prime}}{\varsigma}= & \frac{1}{n+1}\left(f_{2}^{\prime}-f_{1}^{\prime}\right)+\frac{n}{n+1}\left(f_{2}-f_{1}\right) \frac{\varsigma^{\prime}}{\varsigma} \\
& +f_{1}^{\prime}\left(\frac{1}{n+1} f_{2}+\frac{n}{n+1} f_{1}\right) \frac{\varsigma^{\prime}}{\varsigma}=\frac{1}{n+1} f_{2}^{\prime}+\frac{n}{n+1} f_{1}^{\prime} .
\end{aligned}
$$

The contribution of the function $f_{1}$ and $f_{2}$ in Eq. [18] can be captured by one function $h$ which is introduced in Eq. [19]

$$
h(x)=\frac{1}{n+1} f_{2}(x)+\frac{n}{n+1} f_{1}(x)
$$

Equations [18] and [19] yield in following differential Eq. [20] for $h$ as a function of $\varsigma$ and its derivative

$$
\frac{\varsigma^{\prime}}{\varsigma}=\frac{h^{\prime}}{h} \text {. }
$$

The solution for $h$ is given in Eq. [21].

$$
h(x)=\frac{1}{n+1} f_{2}(x)+\frac{n}{n+1} f_{1}(x)=C \varsigma(x)
$$

Equation [21] defines a relationship between the functions $f_{1}$ and $f_{2}$. When Eq. [2] is considered, the function $\varsigma(x)$ varies from $\frac{1}{e}$ to $\frac{1}{s}$. If the constant $C$ is equal to $e v_{\text {IN }}$, which is necessary to fulfill the mathematical translation of condition (i) for the expression for the initial velocity as given in Eq. [11], and the condition for the final velocity of Eq. [11] is fulfilled, Eq. [21] defines an extra relationship between $v_{\text {IN }}$ and $v_{\text {OUT }}$, given in Eq. [22]:

$$
e v_{\mathrm{IN}}=s v_{\mathrm{OUT}}
$$

Condition 22 between the initial and final velocities is automatically fulfilled, since it expresses again the material's incompressibility (condition ii) in the deformation zone.

An assumption of the difference in $\mathrm{x}$ component of the velocity between surface and middle of the sheet, $\left(f_{2}-f_{1}\right)(x)$, is expressed by Eq. [23]:

$$
f_{2}-f_{1}=\alpha\left(e v_{\text {IN }}-f_{1, \text { ref }}\right)=\alpha \Delta f_{\text {ref }}
$$

The parameter $\alpha$ controls the difference between the $x$-component of the velocity between the top surface and the middle of the plate. Higher values of $\alpha$ correspond to higher differences between the functions $f_{1}$ and $f_{2}$. The parameter $\alpha$ has a monotonous relationship with the frictional conditions since more friction will cause larger differences in the velocity along the rolling direction between the mid-thickness and the surface of the sheet. The parameter $\alpha$ is a function of the material's parameters, and technological parameters such as temperature and frictional conditions. The reference function $f_{1 \text {,ref }}$ introduced in Eq. [23] represents the $x$-component of the velocity in the middle of the plate. This component is smaller compared to zero friction $x$-component of the velocity, namely $e \zeta(x) v_{\mathrm{IN}}$, and it becomes larger after the neutral point is reached. The function $f_{1, \text { ref }}$ is defined in Eq. [24].

$$
f_{1, \mathrm{ref}}(x)=\left[1-e^{-a\left(\frac{x}{d}\right)^{b}}\right]\left(v_{\mathrm{OUT}}-v_{\mathrm{IN}}\right)+v_{\mathrm{IN}} .
$$

The parameter $b$ is chosen to be equal to 3 in order to have the reference function of Figure 3. A higher value for $b$ increases the absolute value of $\Delta f_{\text {ref }}$, whereas a lower value for $b$ decreases the difference with the outcome velocity at $x=d$. Since the difference between the middle and the outer velocity is controlled by the 
parameter $\alpha$, see Eq. [23], the choice for $b$ is somewhat arbitrary, as long as it is positive, and not too small. The parameter $a$ is chosen such that $\Delta f_{\text {ref }}$ is zero at a certain value for $x^{*}$, and represents the $x$-value of the neutral point. It is given by Eq. [25].

$$
a=-\left(\frac{d}{x^{*}}\right)^{b} \ln \left(1-\frac{v_{\mathrm{IN}}}{\Delta v}\left(e \zeta\left(x^{*}\right)-1\right)\right)
$$

In this analysis, $x^{*}$ is chosen to be at the half of the deformation zone. The functions $v_{\mathrm{IN}} e \zeta$ and $f_{1, \text { ref }}$ are shown in Figure 3.

Based on the expression for $h$ (Eq. [21]) and for $f_{2}-f_{1}$ (Eq. [23]), the functions $f_{1}$ and $f_{2}$ can be determined.

The $x$ component of the velocity for several values of the parameter $\alpha$ for the current rolling process at the middle layer $\left(f_{1}\right)$, and the surface layer $\left(f_{2}\right)$ are plotted in Figure 4.

At the contact surface, the initial and final velocities (function $f_{2}$ ) are identical, and are initially higher and then lower than the velocities in the middle of the plate (function $f_{1}$ ) because of the frictional conditions, which explains the observed profile of a deformed rectangular grid, put on a plate before the process. The higher the

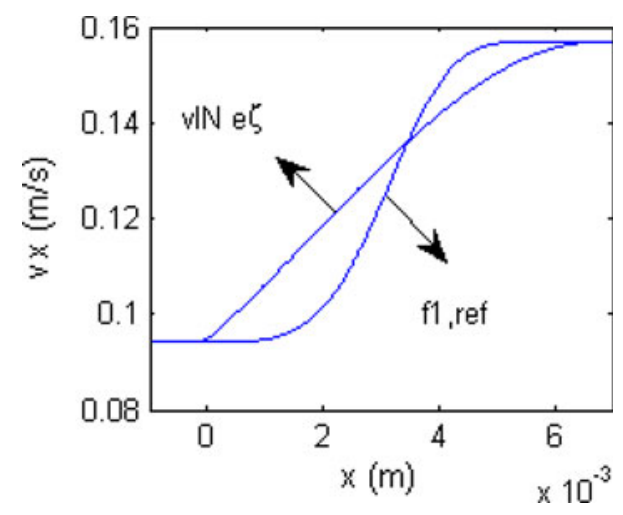

Fig. 3-The reference function $f_{\text {ref }}$, together with the function $e v_{\text {IN }} \zeta(x)$ as a function of the $x$-position in the deformation zone. The function $e v_{\mathrm{IN}} \zeta(x)$ represents the $x$-component of the velocity when there is no difference between the outer and middle layer of the plate.

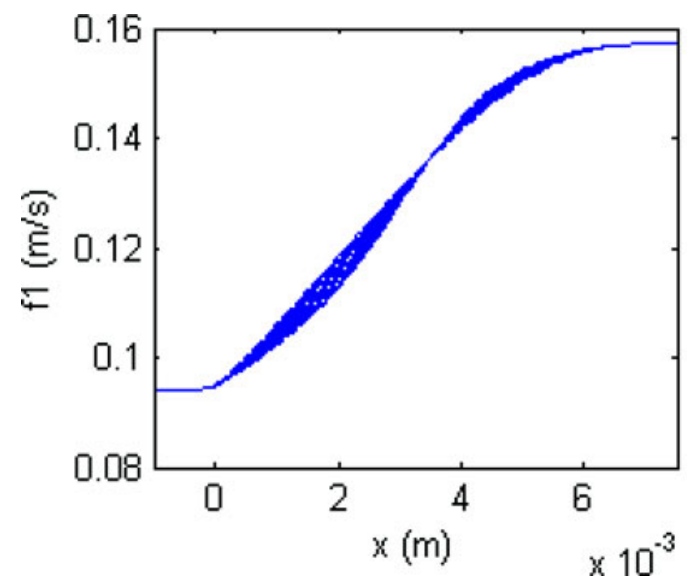

value for $\alpha$, the higher the difference in velocity between middle and top.

The expressions for the velocity as a function of $x$ and $z_{\mathrm{s}}$ of Eqs. [10] and [15], fulfilling all the proposed conditions, is thereby completely determined. Based on the analytical expressions for $v_{x}$ and $v_{z}$, the velocity gradient tensor can be calculated.

\section{Final deformation}

In the deformation zone, except for the beginning and the end, there is a through-thickness gradient in the $x$ component of the velocity. That leads to experimentally observed deformations of an initially rectangular grid brought on the plate as observed for example in the research of $\mathrm{Ji}$ et al. ${ }^{[30]}$ The distortions of the FE mesh for the current rolling process have been calculated by a conventional FE model in Deform 2D ${ }^{\circledR}$ for several linear coulomb friction coefficients are shown in Figure 5.

The simulation of rolling was performed with roll diameter of $R=120 \mathrm{~mm}$, yield point of the material of $380 \mathrm{MPa}$ and Poisson's ratio of 0.3 . Three different friction coefficients of $\mu=0.1, \mu=0.15, \mu=0.25$ were chosen. Although a simplified coulomb friction was considered, Figure 5 shows that the final deformation of an initially rectangular grid is strongly dependent on the frictional conditions between the rolls and the surface of the sheet. In previous section, a method has been introduced to characterize the through-thickness velocity gradient by means of the parameter $\alpha$. Equation [23] shows that the higher the value for $\alpha$, the higher the velocity difference between $f_{1}$ and $f_{2}$, and the higher the velocity gradient over the plate's thickness. A higher velocity gradient leads to a more extensive distortion of the grid.

Two material points of the plate with the same $x$-coordinate but a different $z$ position, before the deformation zone will have a difference in $x$-coordinate after deformation given in Eq. [26]

$$
\Delta x=v_{\mathrm{OUT}}\left(t_{2}-t_{1}\right)
$$

Hereby $t_{1}$ and $t_{2}$ are the times for material point 1 and 2 respectively to pass the deformation zone. The

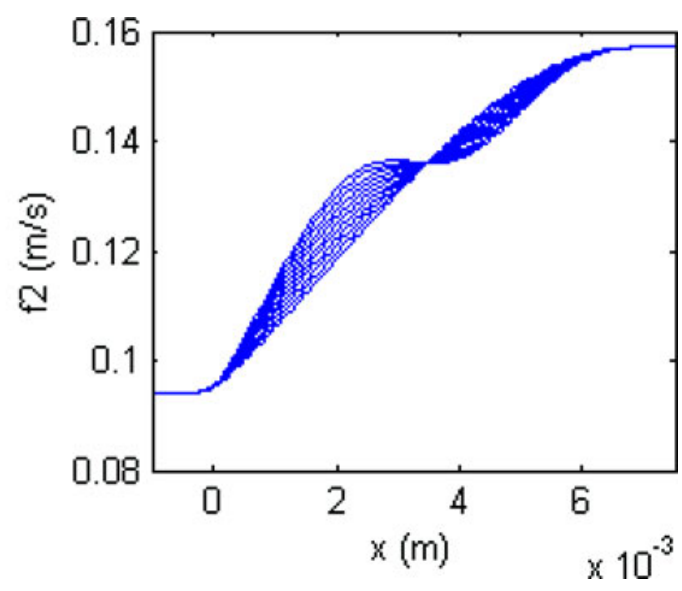

Fig. 4 - The function $f_{1}$ (left-hand side) and $f_{2}$, (right-hand side), defining the $x$-component of the velocity at the middle and the surface for several values for the parameters $\alpha$ from 0 to 1 in steps of 0.1 . The parameter $n=2$. 

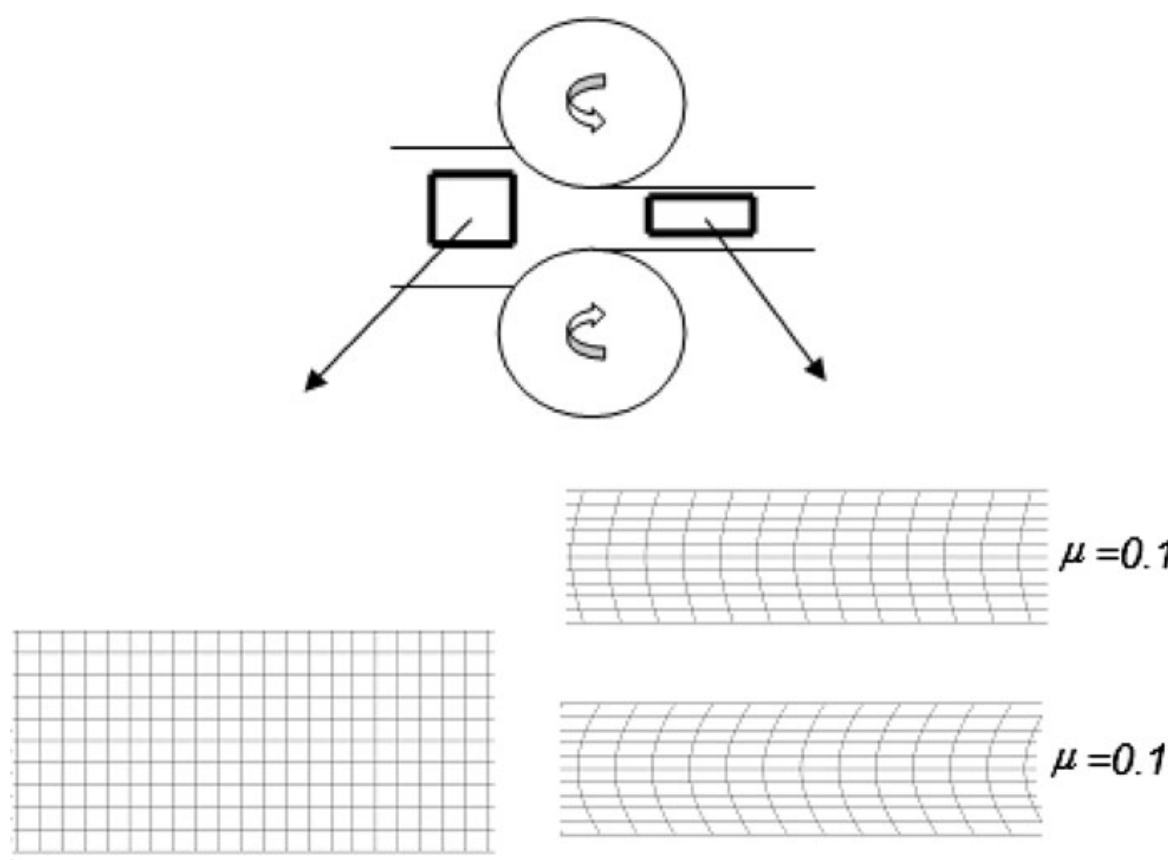

$\mu=0.15$

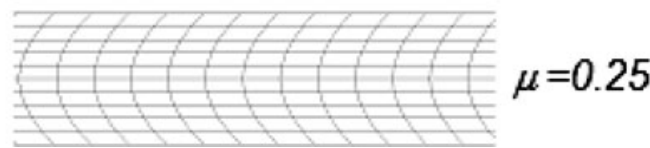

Fig. 5-Deformation patterns of initially rectangular grid emerged after 40 pct thickness reduction as predicted by conventional finite element code Deform 2D ${ }^{\circledR}$ for various friction conditions.

difference in final $x$-value $\Delta x$ between 2 points is given by Eq. [26], since from the time the fastest point leaves the deformation zone, it moves a time $t_{2}-t_{1}$ at a velocity $v_{\text {OUT }}$ until also the second point leaves the deformation zone. The final distortion of an initially vertical line can be expressed by the function $\Delta x\left(z_{\mathrm{INI}}\right)$, which gives the difference in time to pass the deformation zone for all initial values of $z$, defined as $z_{\text {INI }}$, compared to a point at the middle of the sheet. The function $\Delta x\left(z_{\mathrm{INI}}\right)$ is defined as in Eq. [27].

$$
\Delta x\left(z_{\mathrm{INI}}, n, \alpha\right)=v_{\text {OUT }}\left[\int_{0}^{d} \frac{\mathrm{d} x}{v_{x}\left(x, z_{\mathrm{s}}=0\right)}-\int_{0}^{d} \frac{\mathrm{d} x}{v_{x}\left(x, z_{\mathrm{s}}\right)}\right] .
$$

In the first integral of Eq. [27], the coordinate $z_{\mathrm{S}}$ is not a constant, since the flowlines are not exactly followed. The integral for the time, together with the final $\mathrm{z}$ coordinate, can be calculated numerically, by dividing the deformation zone in $\mathrm{N}$ small steps $\Delta x$, and following the procedure presented below.

$$
\begin{aligned}
& x_{1}=0 \\
& z_{1}=z_{\mathrm{IN}} .
\end{aligned}
$$

Loop over $\mathrm{N}$ steps

$$
\begin{aligned}
\Delta x & =\frac{d}{N} \\
x_{i} & =x_{i-1}+\Delta x \\
\Delta z_{i} & =\frac{v_{z}\left(x_{i-1}, z_{i-1}\right)}{v_{x}\left(x_{i-1}, z_{i-1}\right)} \Delta x \\
z_{i} & =z_{i-1}+\Delta z_{i} \\
\Delta t_{i} & =\frac{\Delta x}{v_{x}\left(x_{i-1}, z_{i-1}\right)} \\
t_{i} & =t_{i-1}+\Delta t_{i}
\end{aligned}
$$

End

$$
\begin{aligned}
z_{\mathrm{OUT}} & =z_{\mathrm{N}} \\
t & =t_{\mathrm{N}} .
\end{aligned}
$$

The procedure allows not only to determine the function $\Delta x\left(z_{\mathrm{INI}}, n, \alpha\right)$, but also the function $\Delta x\left(z_{\mathrm{OUT}}, n, \alpha\right)$, the profile of an initially vertical line as a function of the final height, and the friction parameters $n$ and $\alpha$.

Experimentally, one can determine the deformation profile of an initially vertical line as a function of the final $z$-coordinate $z_{\text {OUT }}$, namely $\Delta x_{\mathrm{EXP}}\left(z_{\text {OUT }}\right)$. The friction parameters $n$ and $\alpha$ can be calibrated based on these values that result in a final deformation profile that corresponds the best with the experimentally determined deformation line. The optimal value for $n$ and $\alpha, n_{\mathrm{OPT}}$ and $\alpha_{\mathrm{OPT}}$, can be found with a minimization of the function in Eq. [28] 


$$
\left(n_{\mathrm{OPT}}, \alpha_{\mathrm{OPT}}\right)=\min _{n, \alpha}\left(\int_{z_{\mathrm{FINAL}}=0}^{z_{\mathrm{FINAL}}=e}\left[\Delta x\left(z_{\mathrm{OUT}}, n, \alpha\right)-\Delta x_{\mathrm{EXP}}\left(z_{\mathrm{OUT}}\right)\right]^{2} d z_{\mathrm{OUT}}\right) .
$$

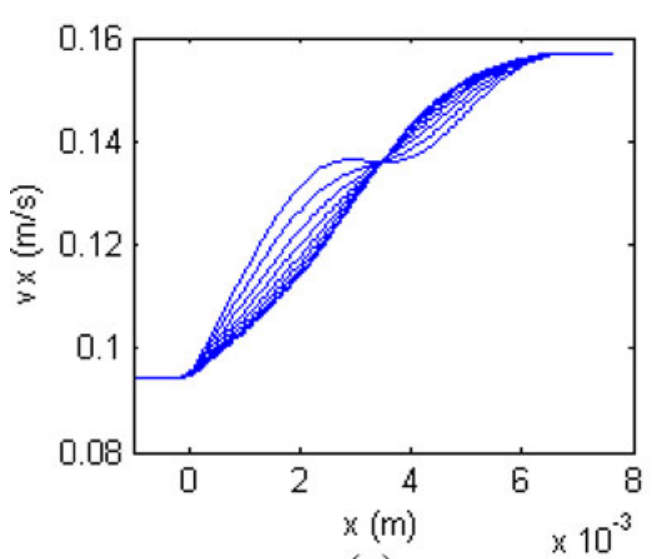

(a)

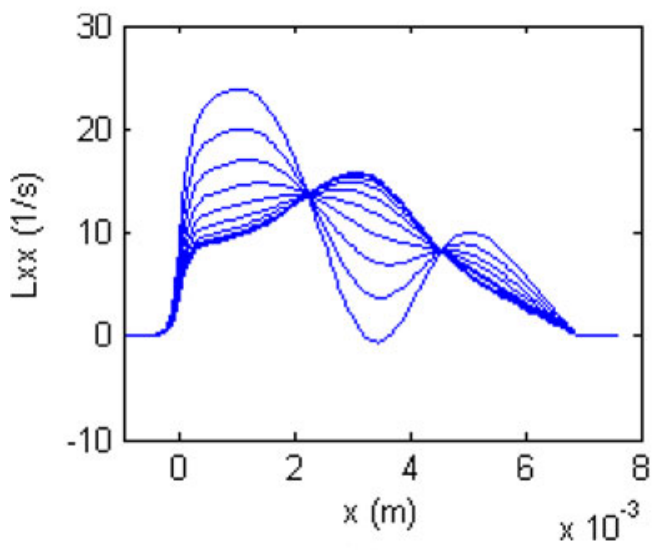

(c)

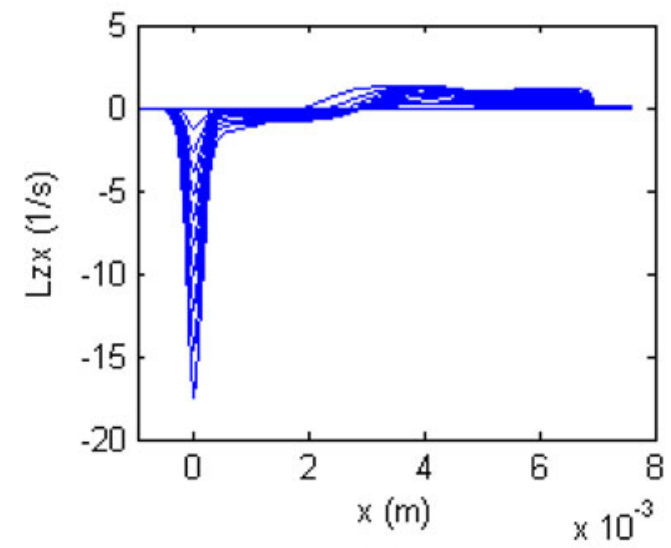

(e)

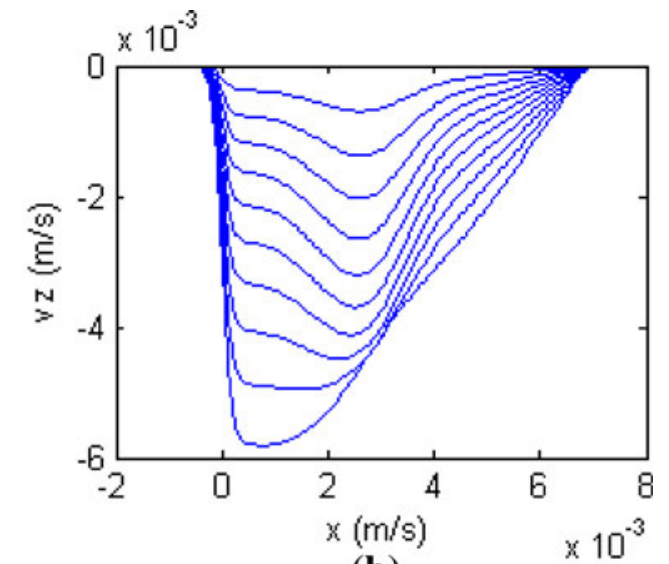

(b)

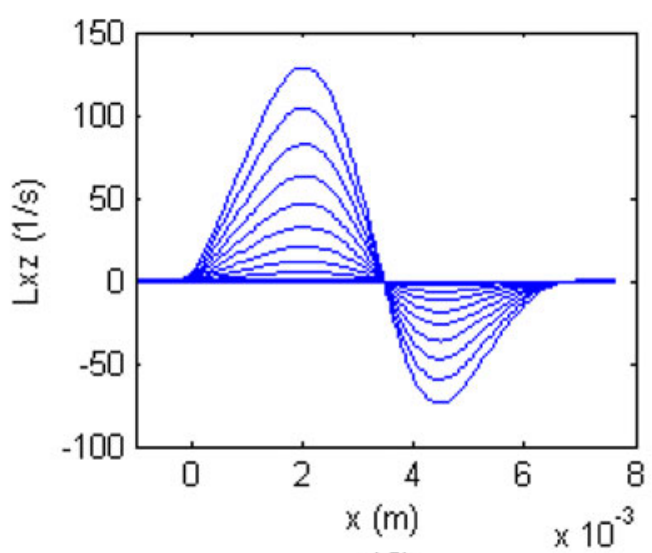

(d)

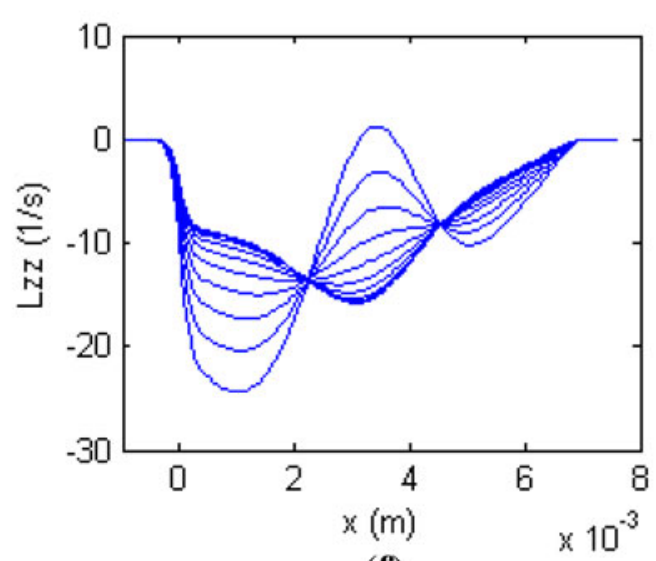

(f)

Fig. 6-Velocity and velocity gradient tensor as a function of the position through the deformation zone $(x)$, for different values of the throughthickness coordinate $\left(z_{\mathrm{s}}\right) . z_{\mathrm{s}}$ varies in steps of 0.1 from 0 to 1 . The value of the parameter $n$ is 2 , and $\alpha=1$. (a) $v_{x},(b) v_{z},(c) L_{x x},(d) L_{x z},(e)$ $L_{z x},(f) L_{z z}$. 


\section{Velocity gradient tensors}

The velocity gradient tensors derived from the velocity field are the input for polycrystal plasticity models which enable calculating the deformation texture and the material's plastic anisotropy. The components of the second rank tensor are given by Eq. [29].

$$
\begin{aligned}
L_{x x}^{*}= & \frac{\partial v_{x}}{\partial x}=\left(f_{2}^{\prime}-f_{1}^{\prime}\right) z_{\mathrm{s}}^{n}+f_{1}^{\prime}+n\left(f_{2}-f_{1}\right) \frac{\varsigma^{\prime}}{\varsigma} z_{\mathrm{s}}^{n} \\
L_{x z}^{*}= & \frac{\partial v_{x}}{\partial z}=\frac{\partial v_{x}^{*}}{\partial z_{\mathrm{s}}} \frac{\partial z_{\mathrm{s}}}{\partial z}=\varsigma\left[n z_{\mathrm{s}}^{n-1}\left(f_{2}-f_{1}\right)\right] \\
L_{z x}^{*}=\frac{\partial v_{z}}{\partial x}=- & \frac{1}{\varsigma}\left[\left(f_{2}^{\prime \prime}-f_{1}^{\prime \prime}\right) \frac{z_{\mathrm{s}}^{n+1}}{n+1}+f_{1}^{\prime} z_{\mathrm{s}}+n\left(f_{2}^{\prime}-f_{1}^{\prime}\right)\right. \\
& \left.\times \frac{z_{\mathrm{s}}^{n+1}}{n+1}\left(\frac{1}{\varsigma}\right)^{\prime}+2\left(f_{2}-f_{1}\right) \frac{z_{\mathrm{s}}^{n+1}}{n+1}\left(\frac{1}{\varsigma}\right)^{\prime \prime}\right] \\
& +\frac{1}{\varsigma^{2}}\left[\left(f_{2}^{\prime}-f_{1}^{\prime}\right) \frac{z_{\mathrm{s}}^{n+1}}{n+1}+f_{1} z_{\mathrm{s}}+n\left(f_{2}-f_{1}\right) \frac{z_{\mathrm{s}}^{n+1}}{n+1} \frac{\varsigma^{\prime}}{\varsigma}\right] \\
= & -\frac{1}{\varsigma}\left[\left(f_{2}^{\prime \prime}-f_{1}^{\prime \prime}\right) \frac{z_{\mathrm{s}}^{n+1}}{n+1}+f_{1}^{\prime} z_{\mathrm{s}}-n\left(f_{2}^{\prime}-f_{1}^{\prime}\right) \frac{z_{\mathrm{s}}^{n+1}}{n+1} \frac{\varsigma^{\prime}}{\varsigma^{2}}\right. \\
& \left.-n\left(f_{2}-f_{1}\right) \frac{z_{\mathrm{s}}^{n+1}}{n+1} \frac{\varsigma^{2} \varsigma^{\prime \prime}-2 \varsigma \varsigma^{\prime 2}}{\varsigma^{4}}\right] \\
& +\frac{1}{\varsigma^{2}}\left[\left(f_{2}^{\prime}-f_{1}^{\prime}\right) \frac{z_{\mathrm{s}}^{n+1}}{n+1}+f_{1} z_{\mathrm{s}}+n\left(f_{2}-f_{1}\right) \frac{z_{\mathrm{s}}^{n+1}}{n+1} \frac{\varsigma^{\prime}}{\varsigma}\right] \\
& \quad L_{z z}^{*}=\frac{\partial v_{z}}{\partial z}=-L_{x x}^{*}
\end{aligned}
$$

Since by construction of Eq. [2], both $\varsigma^{\prime}$ as $\varsigma^{\prime \prime}$ are continuous functions, in the entire region $x \in]-\infty,+\infty[$, all components of the velocity gradient tensor are also continuous during the process.

\section{Texture Prediction}

The $x$-range of the deformation zone is divided in equidistant steps. Per step, the velocity gradient is interpolated at the middle of the interval. The velocity gradient tensor is imposed until the deformation gradient of Eq. [30] is reached

$$
F_{i j}=\delta_{i j}+L_{i j} \Delta t=\delta_{i j}+L_{i j} \frac{\Delta x}{v_{x, i j}}
$$

Hereby the indices $i j$ refer to the elements of the tensors, and $\delta_{i j}$ is Dirac's delta. Per step, an updated Lagrangian technique is used, which implies that at the beginning of the step, the deformation gradient tensor is equal to the unity tensor. The velocity and the velocity gradient tensor are calculated based on the analytical formulation presented in previous section. The VPSC simulation of the texture consists of a sequence of these $\mathrm{N}$ steps. In this paper, 10 steps have been considered.

\section{RESULTS AND DISCUSSION}

\section{A. Velocity Fields and Velocity Gradient Tensor}

Figure 6 shows the velocity and the velocity gradient tensor predicted by the model for the case of the rolling process with geometry presented in Section II, for different values of $z_{\mathrm{s}}$, a value for the parameter $n$ of 2 , and $\alpha$ of 1 .

Note that graphs of Figure 6 are expressed as a function of position, and not as a function of time. The latter can be misleading since the $x$-component of the velocity is increasing with increasing values for $x$, and the time to overcome a given $x$-range is consequently decreasing. The $x z$-component of the velocity gradient tensor changes from positive to negative for the $x$-value where $f_{2}=f_{1}$, which is at the neutral point, being located at $x=d / 2$ in this analysis as mentioned above.

The difference at the outer surface in the $x z$ shear component of the velocity gradient tensor, $L_{x z}$, for different values of the frictional parameter $\alpha$ is shown in Figure 7.

The $L_{x z}$ component shows a sine-shaped type of curve, which was the assumption for shear in study of Engler et al. ${ }^{[18]}$ The sine-shaped function implies that due to the friction, the top layer first moves faster than the underlying layers, and later, when the frictional force works in the opposite direction, this layer moves slower compared to the underlying layers. This effects become more pronounced for higher values of $\alpha$.

In Figure 8 the developed analytical model is compared with the FE simulation of the study of Engler et al. ${ }^{[18]}$ The rolling process considered has a plate reduction from 4.14 to $3.48 \mathrm{~mm}$, with a rolling radius of $127 \mathrm{~mm}$ moving with an angular velocity of 1.5 rotations per minute.

The deformation gradient rates show a good qualitative agreement for all 3 lines considered. In the FE simulations however, the intensities of the peaks of the deformation gradient rates are higher, sometimes up to 3 times. On the other hand, the peaks are broader in the analytical model simulation. Since the ratios of corresponding peak height differences are roughly the reciprocal of the ratios of corresponding peak width

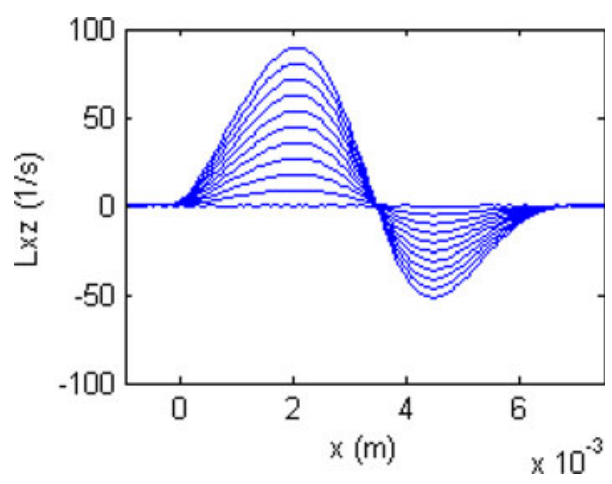

Fig. 7-The $x z$-component of the velocity gradient tensor at the outer surface for different values of $\alpha . \alpha$ is varying from 0 (horizontal curve) to 1 (external curve) in equidistant steps of 0.1 . 

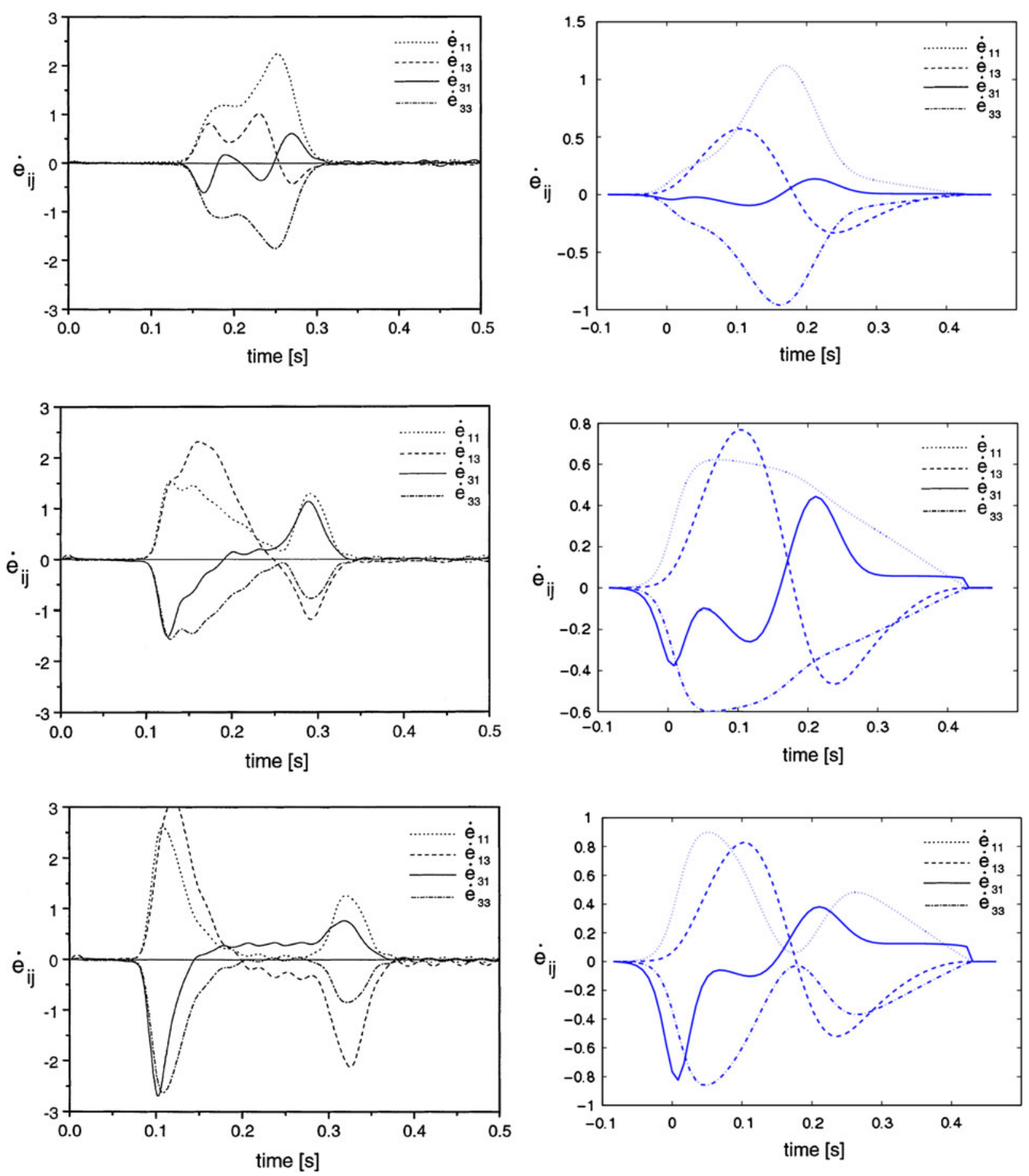

Fig. 8 - Comparison of the deformation gradient rates between a finite element simulation and the analytical velocity model for different places in the sheet. On the left-hand side are the simulations of Engler et al., on the right-hand side the predictions of the analytical velocity model. From upside downwards: $z_{\mathrm{s}}=0.1, z_{\mathrm{s}}=0.5$, and $z_{\mathrm{s}}=0.8$.

differences, the time integrals of the deformation gradient rates, or in other words the final deformation gradients, are similar.

According to the second equation of Formula 29, Figures $6(\mathrm{~d})$ and 7 , the $x z$-(or 13-) shear rate predicted by the model changes sign at the same $x$ coordinate, independent of the through-thickness position. In the FE simulations on the left-hand side of Figure 8, it can be seen that the point in time where the component $\dot{e}_{13}$ changes sign are similar for all values of $z_{\mathrm{s}}$, namely close to 2.5 seconds. Although in the case where $z_{\mathrm{s}}=0.8$ that point is shifted to the left, the value for $\dot{e}_{13}$ remains small around 2.5 seconds. Since in the rolling process considered the relative through-thickness difference in $x$ component of the velocity of the sheet is small, as can be seen in Figure 6(a), similar values for time 
correspond approximately to similar values in $x$-position. Therefore it can be concluded that the model's approximation that the change of $x z$-(or 13) shear rate occurs at the same $x$-position is reasonable. As mentioned in Section III-B-2, in this work that position $x^{*}$ (see Eq. [25]) has been chosen to be at the half of the deformation zone, but it can be defined to be located elsewhere.

\section{B. Material Flow}

When the velocity field is known, the flow of the material can be tracked using the principle described above in Section III-B-3. For 10 equidistant points in the plate, starting from the middle towards the end of the plate, the proposed velocity field has been imposed. The path of the points is shown in Figure 9.

No macroscopic difference in material flow with the flowline model of Figure 2 can be found. For a streamline model determined by Eq. [2], the $z_{\mathrm{IN}}-z_{\text {OUT }}$ relationship between the height of a material point at the entry of the deformation zone and the height at the exit, is determined by Eq. [31]

$$
\frac{z_{\mathrm{IN}}}{z_{\mathrm{OUT}}}=\frac{z(x=0)}{z(x=d)}=\frac{e}{s}
$$

According to Eq. [30], streamline models result in a linear relationship between $z_{\text {IN }}$ and $z_{\text {OUT }}$. For current analytical model, the relationship $z_{\mathrm{IN}}-z_{\text {OUT }}$ has been numerically determined by the scheme presented above, and is plotted for all values of $n$ and $\alpha$ in Figure 10 .

Figure 10 reveals that the relationship $z_{\mathrm{IN}}-z_{\mathrm{OUT}}$ is nearly linear, for all values of $n$ and $\alpha$. Based on Figures 9 and 10, it can be concluded that the material flow deviation from the flowlines in a flowline model of the newly presented model is minimal.

\section{Final Deformation}

The deformation pattern of an originally vertical line is shown in Figure 11 for a wide range of $n$ and $\alpha$ values.

When $\alpha=0$, there is no deformation of an initially vertical line, whereas for $\alpha=2.5$, the difference in $x$-component between the outer surface and the middle of an initially vertical line is $\sim 0.4 \mathrm{~mm}$. All values of $\alpha$ between 0 and 2.5 cover deformations between these 2 limit cases.

\section{Crystallographic Texture}

Polycrystal plasticity models are capable of predicting the deformation texture with high accuracy on the condition that the displacement field is known. The modeled through-thickness textures at three positions of the rolled plate are shown by means of ODF sections in Figure 12

Figure 12(a) accounts for typical plane strain compression texture with characteristic $\alpha$ and $\gamma$ fiber orientations. ${ }^{[1]}$ The $\alpha$ fiber consists of orientations where the $\langle 110\rangle$ direction tends to align along the rolling direction. It includes the orientations $\{001\}\langle 110\rangle$,

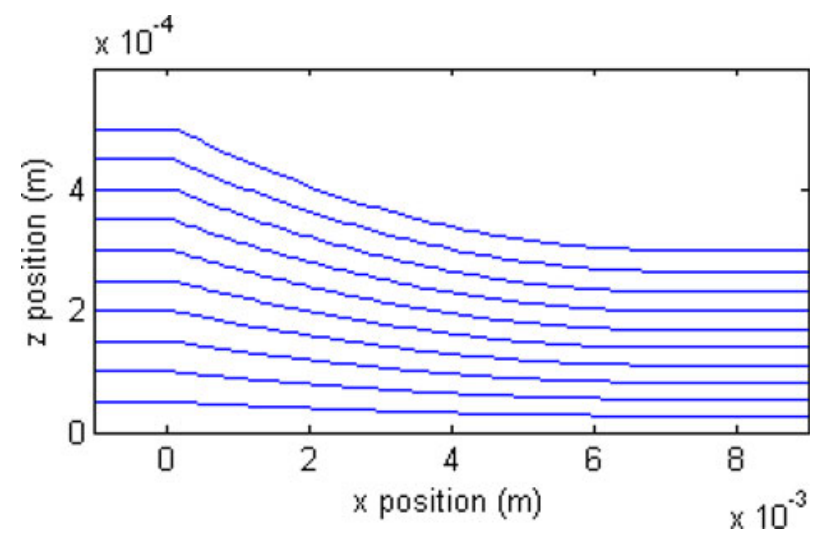

Fig. 9-Material flow according to the new model.

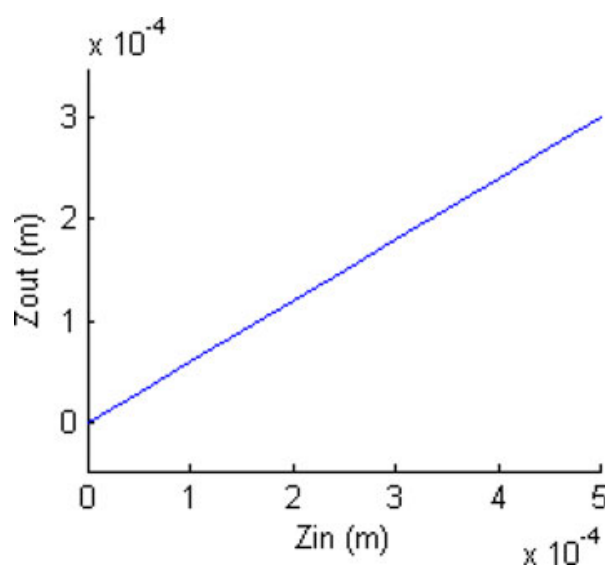

Fig. 10-The initial $z$ value $v s$ the final $z$ value for different values for the parameters $n$ and $\alpha$. The final $z$ value has been calculated by tracking the material flow originating from before the deformation zone at an initial height. $\alpha$ is varying from 0 to 2 , and $n$ is varying from 1 to 5 .

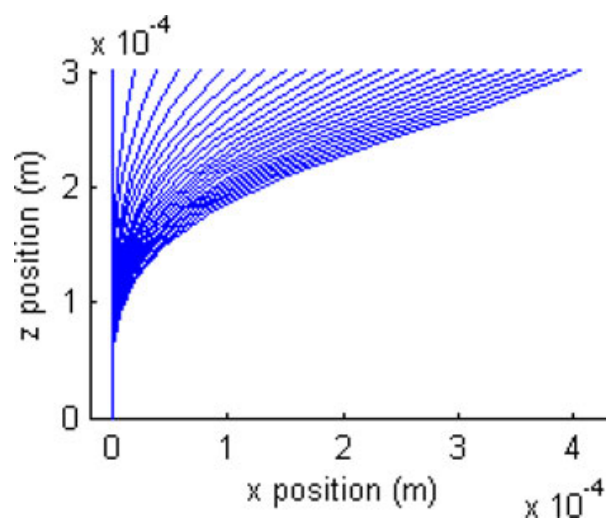

Fig. 11-Deformation profile of an original vertical line for several values of the parameter $\alpha . n=2, \alpha$ is varying from 0 (vertical curve left) to 2.5 (right curve) in steps of 0.15 .

$\{112\}\langle 110\rangle$, and $\{111\}\langle 110\rangle$. The $\gamma$ fiber consists of orientations with the $\{111\}$ planes parallel to ND, such as $\{111\}\langle 110\rangle$ and the $\{111\}\langle 112\rangle$ orientation. The ODF plots at three quarter thickness in Figure 12(b) show 


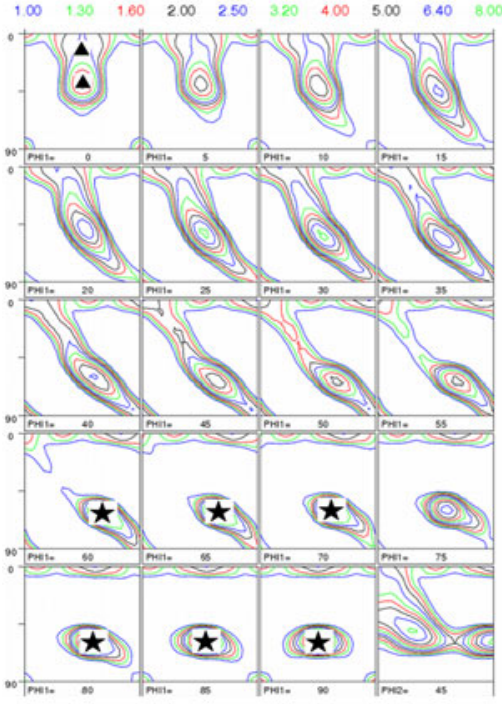

(a)

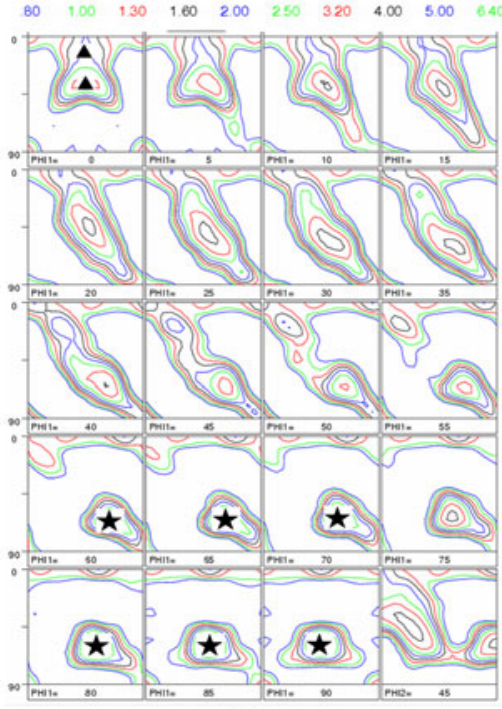

(b)

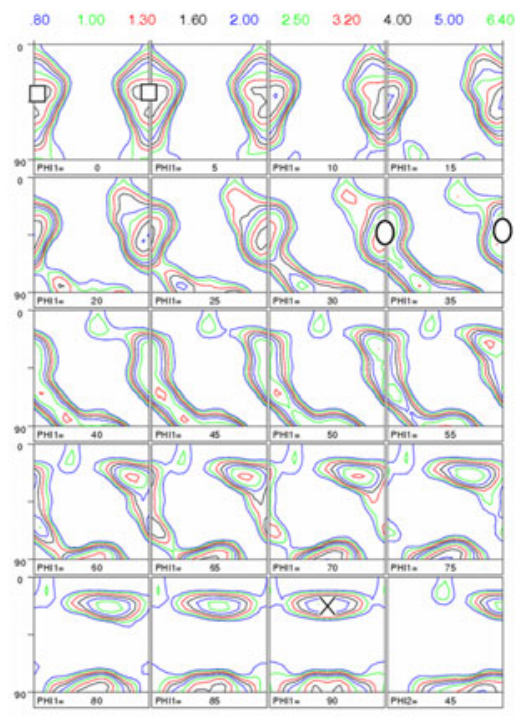

(c)

Fig. $12-$ ODF plots in sections of constant $\varphi_{1}$ in steps of 5 deg for the parameter $\alpha=0.6$, and $n=2$. Fig. 10(a): at middle of the plate. Fig. 11(b): at three quarters of the thickness. Fig. 11(c): at the external surface. The filled triangle refers to the $\alpha$ fiber, the filled star to the $\gamma$ fiber, the square to the $\{011\}\langle 100\rangle$ component, the oval to the $\{011\}\langle 211\rangle$ component, the cross to the $\{112\}\langle 111\rangle$ component.

texture qualitatively identical to one observed at the mid-thickness plane. Figure 12(c) shows a typical shear texture with prevailing Goss component $\{011\}\langle 100\rangle$, the Brass orientation $\{211\}\langle 110\rangle$ and the Copper component $\{112\}\langle 111\rangle$, For $\alpha=0.6$, and $n=2$, the average through-thickness texture is dominated by the $\alpha$ and $\gamma$ fiber orientations. Closer to the surface though, the shear texture components become predominant.

The evolution of crystallographic texture is presented by means of ODF plots along 3 points on a line at the surface, at three quarters, and at the middle of the plate for the intermediate case $\alpha=0.6, n=2$ in Figure 13 . The ODF is represented in $\varphi_{2}=45 \mathrm{deg}$ sections of the Euler space for $\varphi_{1} \in[0,90]$ and $\phi \in[0,90]$. It is important to note that using such a section to represent the entire ODF is only justified when the ODF shows orthotropic symmetry, which is the case only in the middle of the sheet where there is a plane strain compression during the rolling process. The representation of Figure 13 is chosen in order not to overload the figure.

On the top surface, the texture is initially evolving in the direction of a typical plane strain compression texture, to later on break up and evolve to a shear texture. Again, the plots show a similar plane strain compression texture in the middle and at three quarters of the sheet, but with the maximum intensity about 2.5 times higher in the middle.

The influence of different frictional conditions on texture evolution has been investigated by varying the frictional parameter $\alpha$ in the analytical velocity model. The results are shown in Figure 14, where the ODF is plotted in $\varphi_{2}=45 \mathrm{deg}$ sections of the Euler space at the end of the process, and at the three quarter thickness for the cases $\alpha=0.7$ to $\alpha=1.2$, in steps of 0.1 . The parameter $n$ is 2 in all considered cases. The same remark about the section as for Figure 13 should be made.

From Figure 14 it can be concluded that for increasing shear (increasing $\alpha$ ), at mid-thickness the texture evolves from a plane strain compression texture towards a shear texture for higher values for $\alpha$. The limit case is somewhere between 0.8 and 0.9 .

\section{CONCLUSIONS}

A new analytical model is presented that expresses kinematically admissible velocity fields in rolling processes. The model introduces a new coordinate which is constant over the lines that are used as streamlines in streamline models, but does not demand that prescribed streamlines are followed exactly. The model has a prescribed $x$-component of the velocity in the middle of the plate, on the external surface, and both before and after the deformation zone. The effect of shear in the plate is incorporated by introducing 2 frictional parameters. The resulting material flow has shown to have no significant deviation from the corresponding streamlines.

Two model parameters are introduced, namely the parameter $\alpha$ and the parameter $n$. The parameter $\alpha$ controls the difference in velocity between the top and the middle of the plate. The parameter has a monotonous relationship with the frictional conditions. The parameter $n$ controls the distribution of this difference over the thickness of the plate. The parameter $\alpha$ is dependent on the material, the temperature, and the frictional conditions, where the parameter $n$ is dependent only on the material and the temperature. 

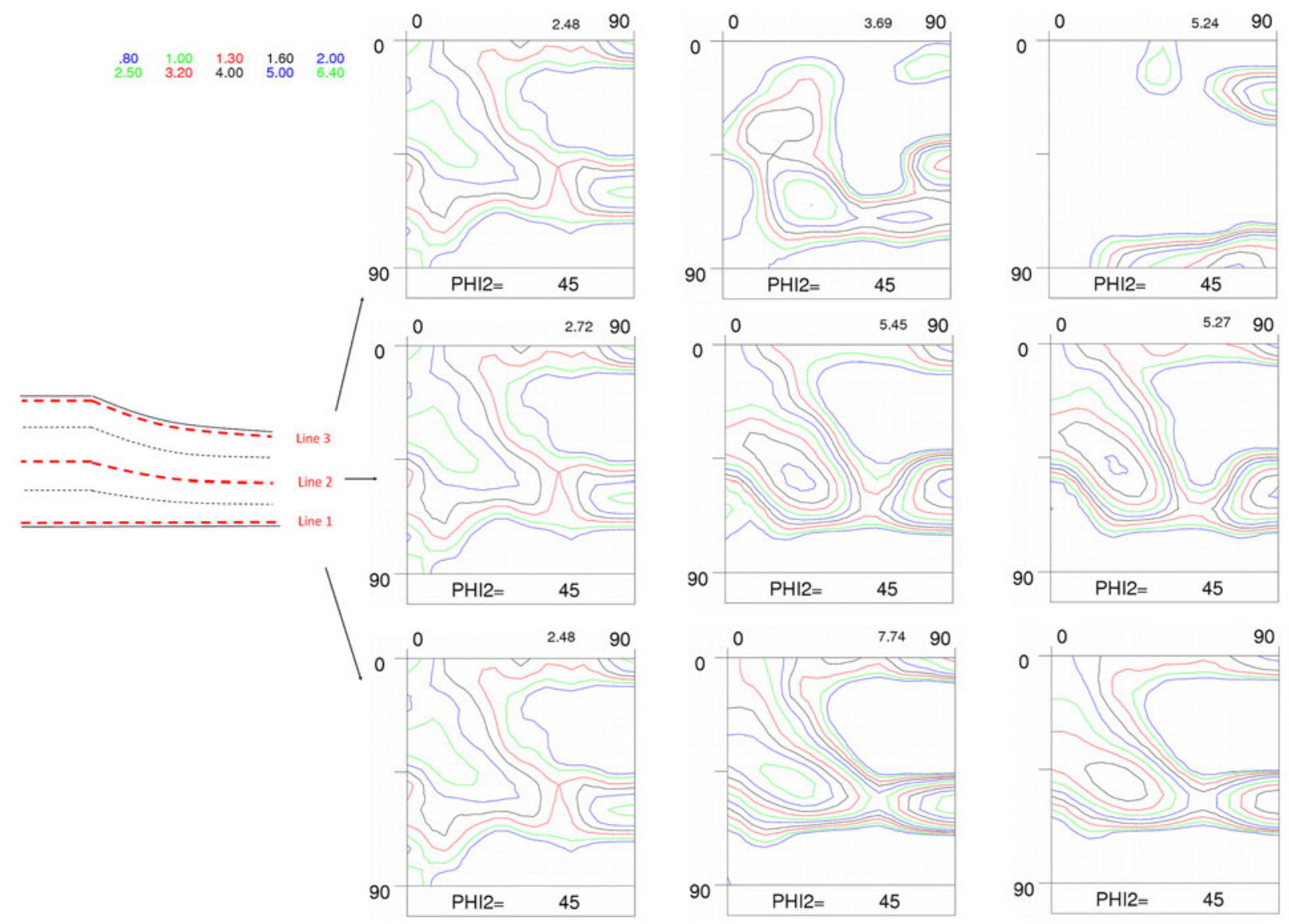

Fig. 13-ODF plots in sections of constant $\varphi_{1}$ in steps of 5 deg for the parameter $\alpha=0.6$, and $n=2$. (a) At middle of the plate, $(b)$ at three quarters of the thickness, $(c)$ at the external surface.
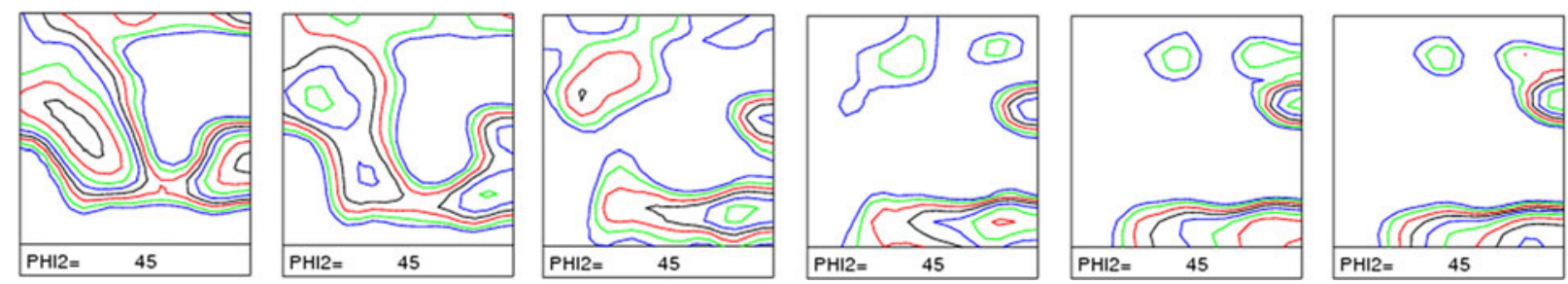

Fig. $14-$ ODF plotted in $\varphi_{2}=45$ deg sections of the Euler space at the external surface. From left to right, $\alpha$ is varying from 0.7 to 1.2 in steps of 0.1 . The parameter $n$ is 2 in all cases.

The model is able to capture the observed throughthickness shear deformation after rolling. The effect of the frictional parameters on the predicted distortion of a grid pattern attached to a plate has been shown. It allows to experimentally determine the values of the frictional parameters by a simple optical experiment for a given rolling process.

The model is able to capture the observed throughthickness shear deformation during rolling. The effect of the frictional parameters on the predicted distortion of a grid pattern has been shown to be able to experimentally determine the values of those parameters by a simple optical experiment for a given rolling process.
The model also provides an analytical expression for the velocity gradient tensor, which can be used for fast texture and anisotropy prediction, when coupling with a polycrystal plasticity model. The results of throughthickness texture predictions for different frictional conditions have been presented. The differences in texture prediction between the centreline and the upper line show that there is a strong through-thickness texture gradient, which is dependent on the frictional conditions. Through-thickness texture measurements can be used to calibrate the model parameters $\alpha$ and $n$.

As the presented model is analytical, it allows a fast calculation of the texture that develops during the rolling process. It could be used to implement plastic 
anisotropy and therefore a more advanced material law in models that calculate rolling forces and torques.

\section{ACKNOWLEDGMENTS}

The authors gratefully acknowledge financial support from the Deutschen Forschungsgemeinschaft (DFG) through Priority Program 1204 "Algorithms for fast Analysis and Simulation in Metal Forming".

\section{REFERENCES}

1. U.F. Kocks, C.N. Tome, and H. Wenk: Texture and Anisotropy: Preferred Orientations in Polycrystals and Their Affect on Materials Properties, Cambridge University Press, Cambridge, 1998, p. 692.

2. S. Kobayashi and T. Altan: Metal Forming and the Finite Element Method, Oxford University Press, Oxford, 1989, p. 396.

3. T. von Karaman: Z. Angew. Math. Mech., 1925, vol. 5, pp. 130 41

4. R.G. Fenton and H.A. Khataan: in Process Modelling: Fundamentals and Applications to Metals, T. Altan, ed., ASM, Cleveland, OH, 1980, p. 448.

5. H. Takuda, N. Hatta, H. Lippmann, and J. Kokado: Ing. Arch., 1989, vol. 59, pp. 274-84.

6. H. Sheikh: Appl. Math. Model., 2009, vol. 33, pp. 2187-95.

7. K. Komori: Int. J. Mech. Sci., 2002, vol. 44, pp. 37-55.

8. L. Anand and S.R. Kalidindi: Mech. Math., 1994, vol. 17, pp. 223 43.

9. L. Delannay, P.J. Jaques, and S.R. Kalidindi: Int. J. Plast., 2006, vol. 22, pp. 1879-98.

10. G. Taylor: J. Inst. Met., 1938, vol. 62, pp. 307-24.

11. R.A. Lebensohn and C.N. Tome: Acta Metall. Mater., 1993, vol. 41, pp. 2611-24.
12. P. Van Houtte, S. Li, and L. Delannay: Int. J. Plast, 2005, vol. 21, pp. 589-624.

13. M. Crumbach, G. Pomana, P. Wagner, and G. Gottstein: in Recrystallisation and Grain Growth, Proc. First Joint Conf., G. Gottstein and D.A. Molodov, eds., Springer, Berlin, 2001, p. 1408.

14. Q. Xie, P. Eyckens, H. Vegter, J. Moerman, A. Van Bael, and P. Van Houtte: Mater. Sci. Eng. A, 2013, vol. 581, pp. 66-72.

15. C.H. Choi, J.W. Kwon, K.H. Oh, and D.N. Lee: Acta Mater., 1997, vol. 45, pp. 5119-28.

16. P. Van Houtte, J. Gawad, P. Eyckens, B. Van Bael, G. Samaey, and D. Roose: Proced. IUTAM, 2012, vol. 3, pp. 67-75.

17. J. Segurado, R.A. Lebensohn, J. LLorca, and C.N. Tomé: Int. J. Plast., 2012, vol. 28, pp. 124-40.

18. O. Engler, M.Y. Huh, and C.N. Tomé: Metall. Mater. Trans. A, 2000, vol. 31A, pp. 2299-2315.

19. A.N. Dogruoglu: J. Mater. Proc. Technol., 2001, vol. 110, pp. 287-99.

20. S.I. Oh and S. Kobayashi: Int. J. Mech. Sci., 1975, vol. 17, pp. 293-305.

21. S.H. Zhang, D.W. Zhao, and C.R. Gao: Int. J. Mech. Sci., 2012, vol. 57 , pp. $74-78$.

22. D. Zhao, S. Zhang, C. Li, H. Song, and G. Wang: J. Iron Steel Res. Int., 2012, vol. 19, pp. 20-24.

23. S. Sezek, B. Aksakal, and Y. Can: Mater. Des., 2008, vol. 29, pp. 584-96.

24. J.P. Wanga, W.C. Kao, H.D. Lee, and J. Wang: J. Mater. Process. Technol., 2008, vol. 204, pp. 425-33.

25. B. Beausir and L.S. Toth: Mater. Sci. Forum, 2005, vols. 495-497, pp. $1603-08$.

26. B. Beausir and L.S. Toth: in International Conference on Microstructure and Texture in Steels, A. Haldar, S. Suwas, and D. Bhattacharjee, eds., MATS2008, Springer Verlag, Jamshedpur, India, 2008, p. 488.

27. S. Li, F. Sun, and H. Li: Acta Mater., 2010, vol. 58, pp. 1317-31.

28. C. Boldetti, C. Pinna, I. Howard, and G. Gutierrez: Appl. Mech. Math., 2005, vols. 3-4, pp. 111-16.

29. H. Valberg: Applied Metal Forming Including FEM Analysis, Cambridge University Press, Cambridge, 2010, p. 460.

30. Y.H. Ji and J.J. Park: Mater. Sci. Eng. A, 2009, vol. 499, pp. 14 17. 